\title{
A Novel Technique to improve the Performance of Wireless Sensor Network using Adaptive Antennas and High-Altitude Platform Communications
}

\author{
Yasser Albagory \\ Taif University, \\ College of Computers \& \\ Information Technology,
}

Taif, Saudi Arabia

\author{
Mostafa Nofal \\ Taif University, \\ College of Computers \& \\ Information Technology, \\ Taif, Saudi Arabia
}

\author{
Omar Said \\ Taif University, \\ College of Computers \& \\ Information Technology, \\ Taif, Saudi Arabia
}

\begin{abstract}
In this paper, the performance of Wireless Sensor Networks (WSN) is improved using adaptive antenna technique and High-Altitude Platforms Systems (HAP). An adaptive concentric circular array (ACCA) is proposed to improve the communications link between sink and sensor nodes. The system is first demonstrated for several scenarios including different cell sizes at a HAP height of $20 \mathrm{~km}$ and the quality of link in terms of the ratio of bit energy to noise power is demonstrated where it shows the capability and reliability of building HAP-WSN despite of the long distance between ground sensors and HAP sink. The proposed ACCA technique provides a power gain profile that both increases the power to and from sensor nodes as well as it reduces the out-of cell radiation to other HAP-WSN areas.
\end{abstract}

\section{Keywords}

HAP, WSN, Adaptive Antenna, Concentric Circular Arrays

\section{INTRODUCTION}

The huge advances in wireless communications technologies have aided other related systems to gain more interest and provide several innovative services. Recently, Wireless Sensor Network (WSN) has gained attention where it is applied in many applications including vital industrial manufacturing techniques, monitoring in military applications, agriculture and fluid detection, and in several security issues [1-4]. This network contains a number of remote sensors that measure the physical quantities and forward its data to a central point called sink station. For ground WSN systems, the distance between sensors and sink points depends on the communication environment where the power exponent is almost around 4 . The other main parameter in WSN is the power source available for sensors which usually comes from batteries and forms a very limitation to the system lifetime. Another main issue regarding WSN is the extension of radio coverage which depends on the communication technique applied between these sensor nodes and sinks and the antenna will play an important parameter for both sensors and sink power drains reduction. The groundbased WSN has a very limited communication range due to the channel impairments including multipath fading problems. The existing ground based WSN has limited its cell coverage to few meters due to the limited battery life time at sensors. The coverage range and battery life time can be extended by switching to a different type of propagation channel to reduce the multipath fading problem namely using the free-space or line-of-sight communications. This kind of propagation channels can be obtained by applying satellite communications or simply using very high sink station that may be located on a very high mast or tower. Recently, an efficient and reliable communication system based on the High-Altitude Platform (HAP) [5-8] has gained interest in research for WSN and has been proved for the existing sensors technologies without using extra sensor power while the coverage radius may extend to several tenses of kilometers. HAP is an aerial platform that operates in a quasistationary position in the stratospheric layer at altitudes ranging from $20-50 \mathrm{~km}$ high and carrying communications payloads [9-16]. The communication link performance of the HAPWSN can be improved by configuring the antennas at the HAP where the antenna technology plays a very important rule [17-28].

The paper is arranged as follows; section 2 describes the HAPWSN system configuration and section 3 provides the communications link equations that describe HAPWSN. Section 4 proposes the adaptive antenna technique for HAPWSN coverage as well as the conventional antenna and section 5 provides simulation results for both the conventional antenna and the proposed antenna array technique. Finally section 6 concludes the paper.

\section{HAPWSN CONFIGURATION}

HAP is an aerial station that is capable to provide a variety of communications applications, monitoring, surveillance, and even in noncommercial military applications. Recently, the HAP provides a good candidate for WSN which may be used in many scenarios. In this paper the HAP acts as a global sink station that collects the ground sensors data either from a large area or in a cellular fashion as shown in Fig. 1. The use of HAP as a sink station provides not only a large area coverage, but also a security on the sink itself as it is highly elevated. In addition, the HAP acts as a global sink station that can provide coverage up to $1000 \mathrm{~km}$ diameter when located at 20 $\mathrm{km}$ altitude. The structure shown in Fig. $1 \mathrm{can}$ be modified to include sub-sink stations on ground which collects data from nearby sensor nodes and forward it to the global HAP sink. This configuration is suitable for cellular WSN but on the other hand is not secure enough as the sub-sinks are located on the ground. The other proposed scheme is direct transmission from ground sensor nodes to the global HAP sink which will be proved in this paper using the same sensor technology and without the need to increase either the transmitted power from them or the transmitting antennas. 
In the following section, we will describe the HAP-WSN link equation that will be useful in evaluating the system performance.

\section{LINK BUDJET FOR HAPWSN}

The quality of link between a HAP sink and the ground sensors depends on the environment where the sensors exist, the elevation angle between the HAP and the sensors or the breadth of the coverage, transmitting frequency, bit rate and the distance of the link [4]. Additional link parameters also apply such as the transmitting power, transmit and receive antenna gains and the atmospheric conditions. To evaluate the system performance we can rely on the bit energy-tonoise power spectral density which is a main parameter affecting the probability of error in the system according to the modulation scheme applied. To determine this ration we may first define the received power at the HAP sensor as follows:

$$
P_{r}=P_{t} G_{s} G_{H} / P_{L}
$$

where $P_{t}$ is the sensor transmitting power, $G_{S}$ is the sensor antenna gain, $G_{H}$ is the HAP antenna gain and $P_{L}$ is the propagation loss between the sensors and the HAP.

The last equation may be expressed in $\mathrm{dB}$ as:

$$
P_{r}[d B]=P_{t}[d B]+G_{s}[d B]+G_{H}[d B]-P_{L}[d B]
$$

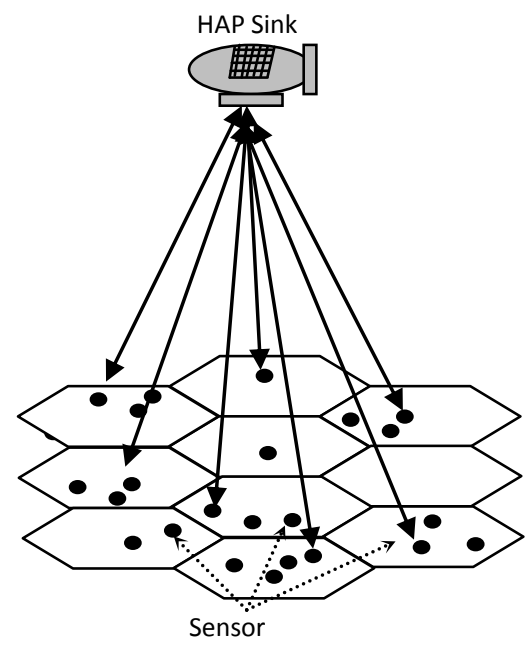

Fig. 1: Cellular HAPWSN

The propagation loss includes both the loss due to the distance and the loss due to shadowing effect:

$$
P_{L}(\mathrm{~d})[\mathrm{dB}]=P_{L}(\mathrm{do})[\mathrm{dB}]+10 n \log \left(d / d_{o}\right)+X_{q}
$$

where $n$ is the pathloss exponent, $d_{o}$ is a reference distance and $d$ in the separation distance between HAP sink and sensor node.

The propagation loss $P_{L}\left(d_{o}\right)$ in $\mathrm{dB}$ is calculated from the following equation:

$$
P_{L}\left(d_{o}\right)=20 \log (4 \pi d / \lambda)
$$

The additional loss $X_{q}$ represents the loss due to the shadowing effects and is characterized as a Gaussian random variable in $\mathrm{dB}$ with zero mean and standard deviation sigma in $\mathrm{dB}$ also.
The value of $\mathrm{n}$ and sigma depend on the propagation environment where for free space propagation $n=2$ and a typical value of sigma in HAPWSN is $2 \mathrm{~dB}$.

The received power at the SP sink is not the only key parameter as a performance measure but another very important quantity denoted as the ration of the bit energy to noise spectral density $\left(E_{b} / N_{o}\right)$ which determines with the modulation scheme the probability of bit error. This ration is given by:

$$
E_{b} / N_{o}(x, y)=P_{t} A_{s} A_{H} / N_{o} R_{b} P_{L}
$$

where $R_{b}$ is the bit rate and (x,y) is the location of the sensor node as shown in Fig. 2(a) which determines the distance d from the following relation:

$$
d=\sqrt{x^{2}+y^{2}+z^{2}}
$$

An example for the coverage of a single HAP at $20 \mathrm{~km}$ high over Taif City, KSA, is depicted in Fig. 2(b) where the whole city can be viewed with very good and high elevation angles that ensure almost line-of-sight propagation scenario. The $E_{b} / N_{o}(x, y)$ may be expressed in $\mathrm{dB}$ as follows:

$$
\begin{array}{r}
E_{b} / N_{o}[\mathrm{~dB}]=P_{t}[\mathrm{~dB}]+G_{s}[\mathrm{~dB}]+G_{H}[\mathrm{~dB}]-P_{L}[\mathrm{~dB}]-N_{o}[\mathrm{~dB}] \\
-R_{b}[\mathrm{~dB}]
\end{array}
$$

As shown from (5) and (7), $E_{b} / N_{o}$ can be improved by increasing the antenna gain either in the transmitting sensor node or at a HAP sink. Increasing antenna gain at the sensor node is physically very difficult due to the increased antenna size and hence the overall sensor volume which in many cases is undesirable. At the HAP sink, the large size and power make it possible to deploy large antennas or antenna arrays which can be optimized to improve the quality of link in HAPWSN as will be discussed in the next section.

\section{ACCA FOR HAPWSN}

In this section, the array configuration and the proposed beamforming technique at the HAP sink will be demonstrated. There are a variety of antenna arrays for beamforming applications such as the planar two-dimensional array, circular arrays and adaptive concentric circular arrays (ACCA) [2942]. The last array configuration has interested features such as the capability of symmetrical beamforming in all azimuth range (i.e. 360 degrees beamforming) with reduced sidelobe levels. In [29-31], the tapered beamforming is applied for ACCA and has proved good performance especially in the sidelobe reduction. The design of flattop ring cells is also demonstrated in [32] where the ACCA is applied using Hamming windows. Other ACCA techniques can be also applied for HAP communications as in [33-42], therefore, in this paper we will use this array configuration to provide both gain and beamwidth requirements.

Assuming that we have $M$ rings ACCA, and then we design a one-dimensional array of $2 M$ elements of a certain sidelobe level $R_{o}$ and obtain the array coefficients from the following design steps:

Find the value of $z_{o}$ from the following equation:

$$
z_{o}=0.5\left(\left(R_{o}+\sqrt{R_{o}^{2}-1}\right)^{1 / p}+\left(R_{o}-\sqrt{R_{o}^{2}-1}\right)^{1 / p}\right)
$$

where $R_{o}$ in this equation is in ratio and $p=2 M-1$ 
The normalized amplitude coefficient of the $m^{\text {th }}$ element in the one-dimensional Dolph-Chebyshev array is calculated as [42]:

$$
\chi_{m}=\frac{\sum_{q=m}^{M}(-1)^{M-q} z_{o}{ }^{2 q-1} \frac{(q+M-2) !(2 M-1)}{(q-m) !(q+m-1) !(M-q) !}}{\sum_{q=1}^{M}(-1)^{M-q} z_{o}{ }^{2 q-1} \frac{(q+M-2) !(2 M-1)}{(q-1) !(q+m-1) !(M-q) !}},
$$

where $m=1,2, \ldots, M$

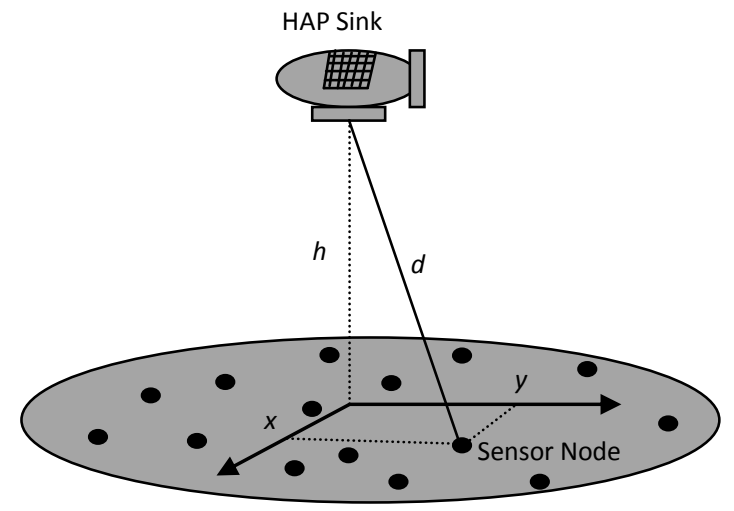

Fig. 2(a): HAPWSN sensor coordinates and link distances.

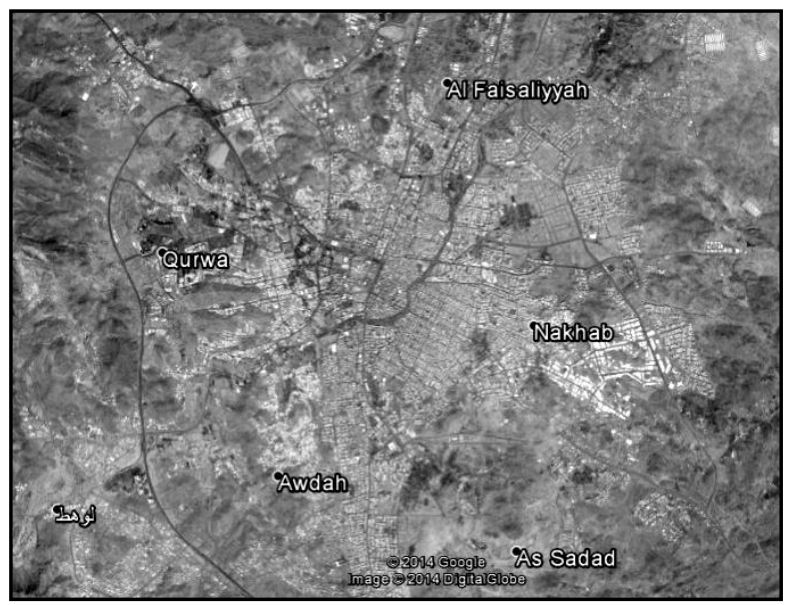

Fig. 2(b): HAP view at $20 \mathrm{~km}$ high over Taif City, KSA.

Finally, the CCA ring coefficients will be given by:

$$
w_{m}=\left(\frac{\sum_{i=1}^{M} N_{i}}{\sum_{i=1}^{M} \chi_{i} N_{i}}\right) \chi_{m}
$$

The resulted sidelobe level of the ACCA using (10) provides sidelobe levels that are different from $R_{o}$ due to the change in the array configuration, but actually when we vary the value of $R_{o}$ the sidelobe levels of the weighted ACCA vary and the sidelobe levels reaches a floor at $R_{o}=80 \mathrm{~dB}$ as demonstrated in [42]. The main purpose here is to provide the required coverage radius by optimizing the array weights, the interelement spacing distances, innermost ring size and the number of rings. The cell boundary can be defined by several limits such as the $3 \mathrm{~dB}$ or $10 \mathrm{~dB}$ power contour. We will apply the $10 \mathrm{~dB}$ contour as the cell boundary as in [23] for the purpose of comparison. The antenna model developed by [18] was adopted where the directivity pattern of the aperture antenna was modeled by:

$$
D(\theta)=(\cos (\theta))^{n} \frac{32 \log (2)}{2(2 \cos (\sqrt[n]{0.5}))^{2}}
$$

Therefore, optimizing this function to find the value of $\mathrm{n}$ will provide a good approximation to the real radiation pattern. The power pattern in (18) is designed for two types of cells; a $30 \mathrm{~km}$ radius and another smaller cell of $8 \mathrm{~km}$ to examine wide coverage and possibility of cellular coverage respectively. The power gain profile for the two cases is shown in Fig. 3 where the first corresponds to the $8 \mathrm{~km}$ cell and the lower curve corresponds to the conventional spotbeam antenna design as in (18) while the second upper curve is for the proposed optimized ACCA that gives the same power profile but at much higher boresight gain.

In addition, Fig. 4 provides the power gain profile for the two antenna types designed for $30 \mathrm{~km}$ HAP cell. In this figure, the optimized ACCA provides an increased higher boresight gain by about $17 \mathrm{~dB}$ which is an incredible amount of power gain difference and should reflect an improvement in the HAPWSN performance.

\section{SIMULATIONS RESULTS}

In this section, four case studies are examined to show the feasibility of the proposed optimized ACCA technique compared to the conventional spot-beam antenna. The comparison is based on the $E_{b} / N_{o}$ as a performance measure of the HAPWSN. The sensor nodes are chosen with the same existing technology. The operating frequency is chosen in the free industrial, scientific and medical (ISM) band. We consider here for comparison the $868 \mathrm{MHz}$ frequency and the transmitting bit rate is $38.4 \mathrm{~kb} / \mathrm{s}$. The modulation scheme is the binary phase shift keying (BPSK). In the first case, as shown in Fig. 5 , the cell radius is $8 \mathrm{~km}$, the transmitting frequency is $868 \mathrm{kHz}$ and $R_{b}$ is $38.4 \mathrm{~kb} / \mathrm{s}$. The two antenna cases show a possible and feasible link between the HAP sink and the ground sensor nodes. In this case, the optimized ACCA shows a better link performance by about $10 \mathrm{~dB}$ due to the improved antenna gain.

The second case is described for the $30 \mathrm{~km}$ cells and the performance comparison is shown in Fig. 6 where it is possible for establishing the network in this case with improved performance for the optimized ACCA. Therefore, in all examined cases, the optimized ACCA provides better performance and feasible link between the HAP sink and the ground sensor nodes although the very long distances separating them. 


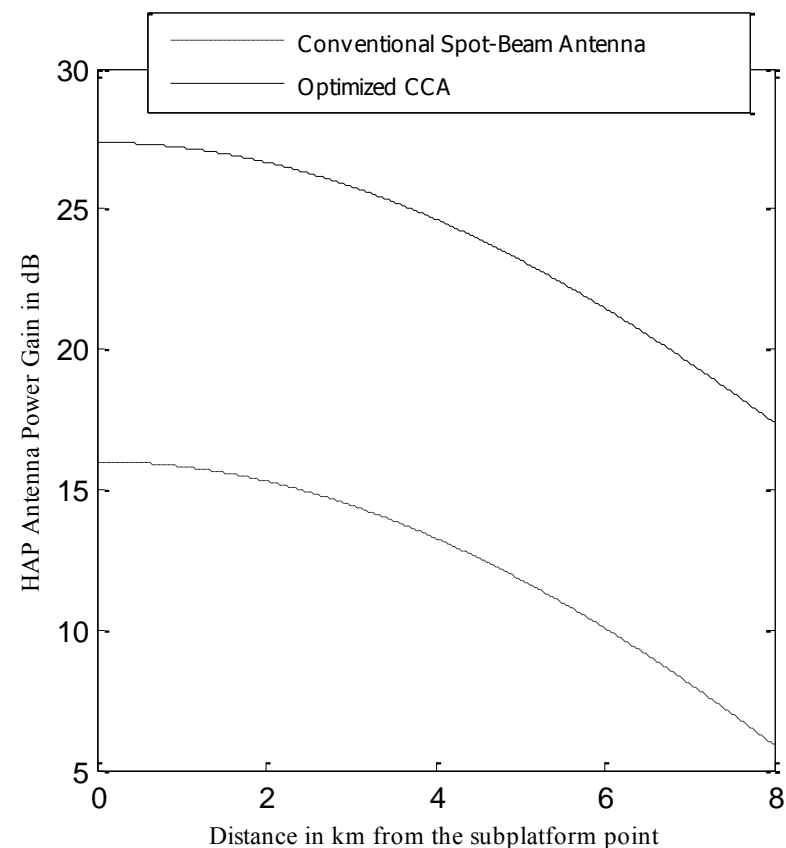

Fig. 3: Gain of conventional antenna and proposed ACCA designed for $8 \mathrm{~km}$ HAP cell.

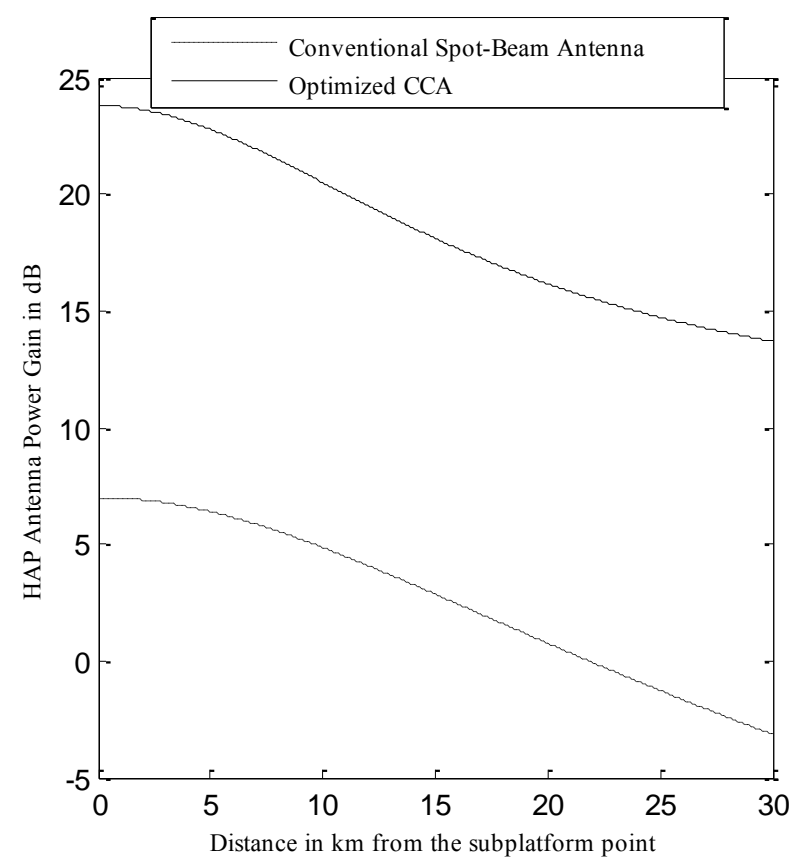

Fig. 4: Gain of conventional antenna and proposed ACCA designed for $30 \mathrm{~km}$ HAP cell.

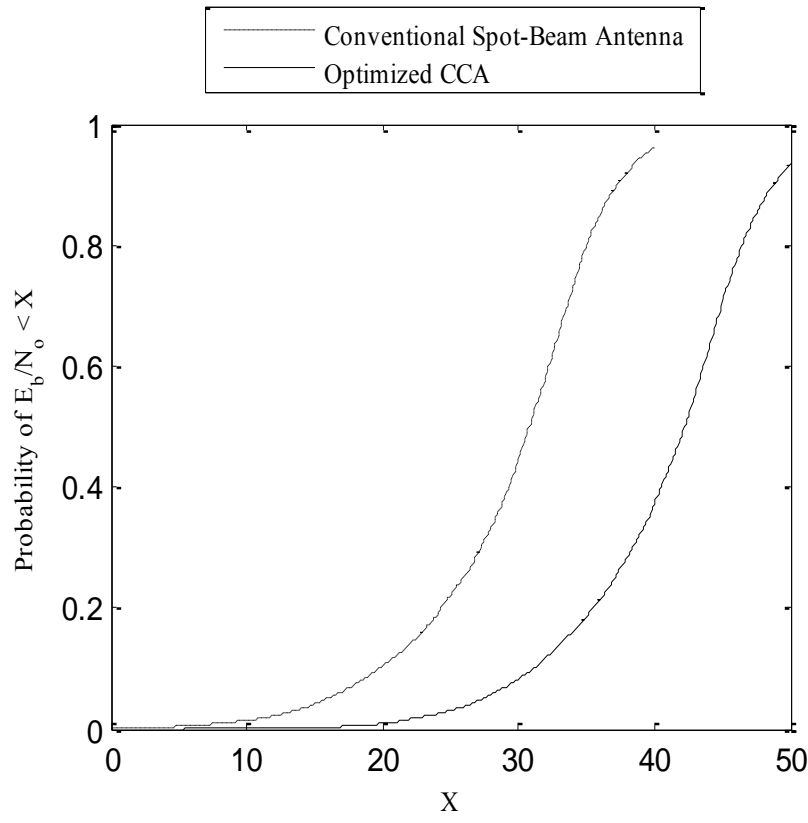

Fig. 5: Eb/No for the two antenna types designed for $8 \mathrm{~km}$ cell at $868 \mathrm{MHz}$ and $\mathrm{Rb}=38.4 \mathrm{~kb} / \mathrm{s}$.

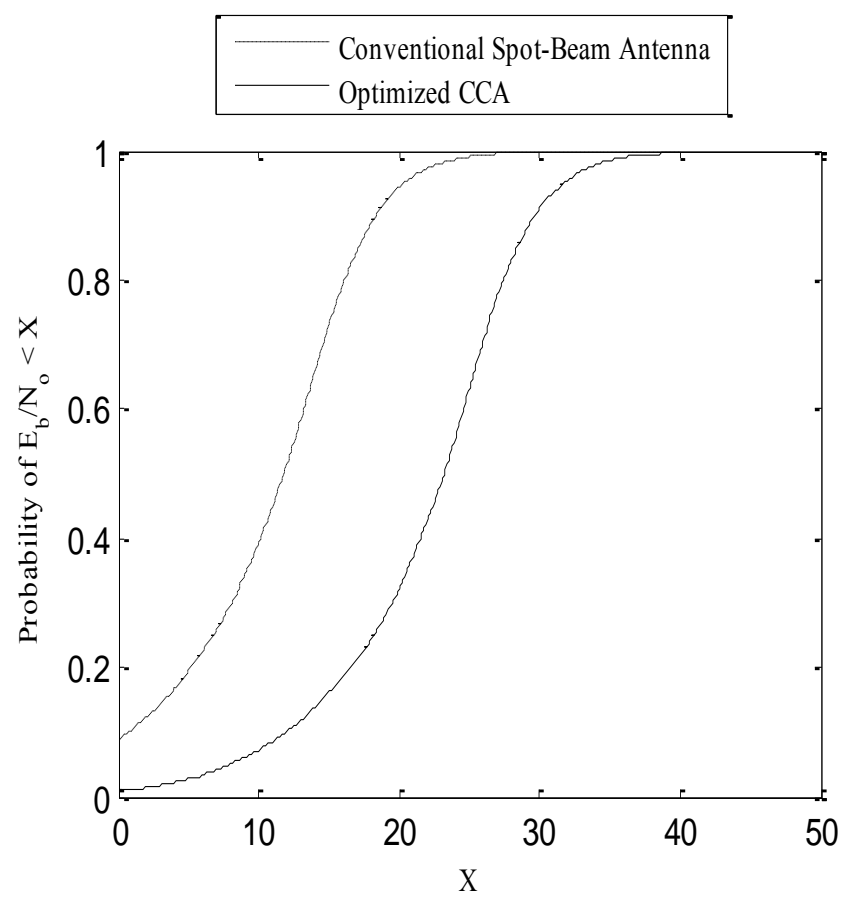

Fig. 6: $\mathbf{E b} / \mathrm{No}$ for the two antenna types designed for 30 $\mathrm{km}$ cell at $868 \mathrm{MHz}$ and $\mathrm{Rb}=38.4 \mathrm{~kb} / \mathrm{s}$. 


\section{CONCLUSIONS}

An efficient WSN based on HAP and adaptive antenna array in the form of ACCA has been demonstrated where the HAP forms a central sink station for collecting nodes data on the ground. The proposed HAPWSN system has provided many advantages over conventional terrestrial or satellite WSN including wide area coverage and superior communications performance by improving the quality of link between the sink and the sensors on the ground. The array is used at the HAP and is optimized to provide better gain and coverage over the required area. The main objective is to minimize the out-of-coverage radiation which is important for frequency reuse cellular WSN.

\section{REFERENCES}

[1] Y. Wang, M. Wilkerson, X. Yu, "Hybrid Sensor Deployment for Surveillance and Target Detection in Wireless Sensor Networks", International Conference on Wireless Communications and Mobile Computing (IWCMC), Istanbul, Turky, pp. 326 - 330, 2011.

[2] R. Alageswaran, R. Usha, R. Gayathridevi, G. Kiruthika, "Design and implementation of dynamic sink node placement using Particle Swarm Optimization for life time maximization of WSN applications", IEEE International Conference on Advances in Engineering, Science and Management (ICAESM), Nagapattin, India, pp. 552 - 555, 30-31 March 2012.

[3] Yasser Albagory, Fahad Al Raddady, Sultan Aljahdali, Omar Said, "Innovative Large Scale Wireless Sensor Network Architecture Using Satellites and High-Altitude Platforms" I.J. Wireless and Microwave Technologies. 03/2014; 4(2):12-19

[4] Yasser Albagory, Omar Said, "Optimizing Concentric Circular Antenna Arrays for High-Altitude Platforms Wireless Sensor Networks", International Journal of Computer Network and Information Security, Vol. 6 No. 5, pp. 1-8, 2014

[5] S. Karapantazis and F. Pavlidou "Broadband Communications via High-Altitude Platforms: A Survey", IEEE Communications Surveys \& Tutorials, First Quarter 2005, pp. 1-31, 2005.

[6] P. Pace and G. Aloi "Disaster Monitoring and Mitigation using Aerospace Technologies and Integrated Telecommunication Networks", IEEE Aeropspace and Electronic Systems Magazine, Vol. 23, No. 4, pp. 3-9, April 2008

[7] Z. Yang, and A. Mohammed, "High Altitude Platforms for Wireless Sensor Network applications", IEEE International Symposium on Wireless Communication Systems 2008, ISWCS2008, pp. 613-617. 2008.

[8] Yasser Albagory, Fahad Alradday," High-Altitude Platforms Cellular System for Sparsely Populated Areas", International Journal of Computer Network and Information Security, Vol. 6 No. 4, pp. 13-20, 2014.

[9] Mostafa Nofal, Yasser Albagory, Mohiy Hadhoud, Moawad Dessouky, "Two-Dimensional Switched-Beam Smart Antenna for Stratospheric Platform Cellular Communication", Proc. Of IEEE International Conference on Software, Telecommunications, and Computer Network (SoftCOM 2001), October 2001.
[10] Yasser Albagory, Moawad Dessouky, Hamdy Sharshar, "Footprint Analysis of High Altitude Platforms Cellular Communications," 2006 China-Japan Joint Microwave Conference (CJMW2006), August 23-25, 2006, Chengdu, China.

[11] Yasser Albagory Moawad Dessouky, Hamdy Sharshar, "Geometrical Analysis of High Altitude Platforms Cellular Footprint," Progress In Electromagnetics Research, PIER 67, pp. 263-274, 2007.

[12] Yasser Albagory, Moawad Dessouky, Hamdy Sharshar, "Design of High Altitude Platforms Cellular Communications," Progress In Electromagnetics Research, PIER 67, pp. 251-261, 2007.

[13] Mostafa Nofal, Yasser Albagory, Mohiy Hadhoud, Moawad Dessouky, "A Novel Cellular Structure for Stratospheric Platform Mobil Communications “, Proc. of the Nineteenth National Radio Science Conference, NRSC'2002, March 19-21, Faculty of Engineering, Alexandria University, Alexandria, EGYPT, 2002

[14] Yasser Albagory, Moawad Dessouky, Hamdy Sharshar, "Improving The Cellular Coverage from A High Altitude Platform by Novel Tapered Beamforming Technique," Journal of Electromagnetic Waves and Applications, JEMWA, Vol.21, No.13, pp. 1721 -1731,2007

[15] Yasser Albagory, "An Efficient Clustering Scheme for High Altitude Platform Mobile Communications", published in the International conference on software, telecommunications and computer networks, SoftCOM 2002, pp. 521-525, 2002

[16] Yasser Albagory, "Investigating the Rotational Motion Effects of the High Altitude Platforms on Mobile Communications", published in the International conference on software, telecommunications and computer networks, SoftCOM 2002, pp. 515-520, 2002.

[17] Yasser Albagory, Moawad Dessouky and Hamdy Sharshar, "Efficient Highways Coverage using High Altitude Platforms", in the 2007 Asia-Pacific Microwave Conference, APMC2007, December 11-14, 2007, Bangkok, Thailand.

[18] Z. Yang, and A. Mohammed, "High Altitude Platforms for Wireless Sensor Network applications", IEEE International Symposium on Wireless Communication Systems 2008, ISWCS2008, pp. 613-617. 2008.

[19] Yasser Albagory, Moawad Dessouky and Hamdy Sharshar, "An Efficient Highways Coverage Technique for the High Altitude Platforms Mobile Communications", has been accepted for publication in the 23rd Progress In Electromagnetics Research Symposium, "PIERS 2008", in Hangzhou, CHINA, 24 28 March, 2008.

[20] Yasser Albagory, Moawad Dessouky and Hamdy Sharshar, "A Novel Wormy Cellular Structure for High Altitude Platforms Mobile Communications", International Journal of Wireless Personal Communications, Vol. 47, pp. 161-169, Springer, 2008.

[21] Yasser Albagory, "A Novel Beamforming Technique for Highways Coverage Using High-Altitude Platforms," Research Letters in Signal Processing, vol. 2007, Article ID 52745, 5 pages, 2007. doi:10.1155/2007/52745. 
[22] Yasser Albagory, "A Novel Design of Arbitrary Shaped Cells for Efficient Coverage from High Altitude Platforms", Progress In Electromagnetics Research Letters, Vol. 1, 245-254, 2008.

[23] H.A. Hossam, Yasser Albagory and S.S. Ehab, "Comparative Study of Different Coverage Models for High Altitude Platform" Menoufia Journal of Electronic Engineering Research, Vol. 19, pp. 29- 47, 2009.

[24] Sultan Aljahdali, Mostafa Nofal and Yasser Albagory, "Overview on Modeling and Design of Stratospheric Mobile Cellular System", IEEE International Conference On Multimedia Computing And Systems (ICMCS'12), Tangier, Morocco: (2012).

[25] Sultan Aljahdali, Mostafa Nofal and Yasser Albagory, "Geometrical Correction for Cell Deployment in Stratospheric Cellular Systems", Progress In Electromagnetics Research C, Vol. 29, pp. 83-96, 2012

[26] Yasser Albagory, "Flat-Top Ring-Shaped Cell Design for High-Altitude Platform Communications", International Journal of Computer Network and Information Security, Vol. 5 No. 7, pp. 51-57, 2013.

[27] Yasser Albagory, "Sectorized Hamming Concentric Circular Arrays for Stratospheric Platforms Cellular Design", International Journal of Computer Network and Information Security, Vol. 5 No. 9, pp. 21-27, 2013

[28] Yasser Albagory, Alaa Abbas, "Smart Cell Design for High Altitude Platforms Communication", AEUInternational Journal of Electronics and Communications, Vol. 67, No. 9, pp. 780-786, September 2013.

[29] Mostafa Nofal, Sultan Aljahdali and Yasser Albagory, "Tapered Beamforming for Concentric Ring Arrays", AEU International Journal of Electronics and Communications, Vol. 67, No. 1, pp. 58-63, 2013.

[30] Sultan Aljahdali, Mostafa Nofal and Yasser Albagory, "A Modified Array Processing Technique Based on Kaiser Window for Concentric Circular Arrays", IEEE International Conference On Multimedia Computing And Systems (ICMCS'12), Tangier, Morocco: (2012).

[31] Mostafa Nofal, Sultan Aljahdali and Yasser Albagory, "Simplified Sidelobe Reduction Technique for Concentric Ring Arrays", Wireless Personal Communications, Vol. 71, No. 4, pp. 2981-2991, Springer, 2013.

[32] Yasser Albagory, "An Approach for Designing Flat-Top High-Altitude Platforms Ring-Shaped Cells Using Vertical Linear Array", Wireless Personal Communications, Vol. 77, No. 1, pp. 661-673, 2014, Springer US.

[33] Basim Mohammed Eldowek, Emmanouel T Michailidis, Yasser A Albagory, Mohamad Abd-Elnaby, El-Sayed M El-Rabaie, Moawad I Dessouky, Abdel-Aziz T Shalaby,
Bassiouny M Sallam, Fathi E Abd El-Samie, Athanasios G Kanatas, "Complex Envelope Second-Order Statistics in High-Altitude Platforms Communication Channels", Wireless Personal Communications, Vol. 77, No. 4, pp 2517-2535, 2014..

[34] Yasser Albagory, "Impact of High-Altitude Platforms Rotation on Cellular Mobile Communications", International Journal of Advanced Computing Research. 03/2014; 1 .

[35] Mostafa Nofal, Yasser Albagory, Moawad Dessouky, Mohiy Hadhoud, "Performance and Feasibility of Different Switched-Beam Antennas for Stratospheric Platform Mobile Communications Covering Newly Developing Regions", Proc. of the Nineteenth National Radio Science Conference, NRSC'2002, March 19-21, Faculty of Engineering, Alexandria University, Alexandria, EGYPT, 2002.

[36] Mostafa Nofal, Yasser Albagory, Moawad Dessouky, Hamdy Sharshar, "Modeling and Investigating the Rotational Motion Effects of the High Altitude Platforms," Twenty Second National Radio Science Conference (NRSC 2005) March 15-17, 2005 Cairo Egypt

[37] Mostafa Nofal, Yasser Albagory, Moawad Dessouky, Hamdy Sharshar, "Optimization of Beams Directions for High Altitude Platforms Cellular Communications Design," Twenty Third National Radio Science Conference (NRSC 2006) March 15-17, 2006, Menouf - Egypt

[38] Yasser Albagory, Moawad Dessouky, Hamdy Sharshar, "A Novel Tapered Beamforming Window for Uniform Concentric Circular Arrays" Journal of Electromagnetic Waves and Applications, JEMWA, Vol. 20, No. 14, pp. 2077-2088, 2006

[39] Yasser Albagory, Moawad Dessouky, Hamdy Sharshar, "Efficient Sidelobe Reduction Technique for SmallSized Concentric Circular Arrays," Progress In Electromagnetics Research, PIER 65, pp. 187-200, 2006.

[40] Yasser Albagory, Moawad Dessouky, Hamdy Sharshar, "Optimum Normalized-Gaussian Tapering Window for Side Lobe Reduction in Uniform Concentric Circular Arrays," Progress In Electromagnetics Research, PIER 69 , pp. $35-46,2007$.

[41] Yasser Albagory, Moawad Dessouky, Hamdy Sharshar, " An Approach for Low Sidelobe Beamforming in Uniform Concentric Circular Arrays" International Journal of Wireless Personal Communications, vol. 43, no. 4, pp. 1363-1368, 2007, Springer.

[42] Yasser Albagory, Moawad Dessouky, Hamdy Sharshar, "An Approach for Dolph-Chebyshev Uniform Concentric Circular Arrays," Journal of Electromagnetic Waves and Applications, JEMWA, Vol.21, No.6, pp.781 $-794,2007$. 